\title{
System integration of hybrid assembled large aperture Micro Scanner Array for fast scanning LiDAR sensors
}

\author{
Thilo Sandner,* Thomas Graßhoff, Wolf-Dietrich Owe, \\ Andreas Herrmann, Michael Wildenhain, \\ Markus Schwarzenberg, and Jan Grahmann \\ Fraunhofer Institute for Photonic Microsystems (IPMS), Department \\ of Active Microoptical Devices and Systems (AMS), Dresden, Germany
}

\begin{abstract}
We present the design and system integration of a hybrid MEMS scanning mirror (MSM) array developed for real-time three-dimensional imaging with a panoramic optical field of view (FOV) of $360 \mathrm{deg} \times 60 \mathrm{deg}$ (horizontal $\times$ vertical). The pulsed time-of-flight light detection and ranging (LiDAR) system targets a distance measurement range of $100 \mathrm{~m}$ with a video-like frame rate of $10 \mathrm{~Hz}$. The fast vertical scan axis is realized by a synchronous scanning MSM array with large receiver aperture. It increases the scanning rate to $3200 \mathrm{~Hz}$, which is four times faster in comparison with state-of-the-art fast macroscopic polygon scanning systems used in current LiDAR systems. A hybrid assembly of frequency selected scanner elements was chosen instead of a monolithic MEMS array to guaranty high yield of MEMS fabrication and a synchronous operation of all resonant MEMS elements at $1600 \mathrm{~Hz}$ with large FOV of $60 \mathrm{deg}$. The hybrid MSM array consists of a separate emitting mirror for laser scanning of the target and 22 reception elements resulting in a large reception aperture of $D_{\text {eff }}=23 \mathrm{~mm}$. All MSM are driven in parametric resonance to enable a fully synchronized operation of all individual MEMS scanner elements. Therefore, piezoresistive position sensors are integrated inside the MEMS chip, used for position feedback of the driving control. We focus on the MEMS system integration including the microassembly of multiple MEMS scanning elements using micromechanical self-alignment. We present technical details to meet the narrow tolerance budgets for (i) microassembly and (ii) synchronous driving of multiple MEMS scanner elements. () The Authors. Published by SPIE under a Creative Commons Attribution 4.0 International License. Distribution or reproduction of this work in whole or in part requires full attribution of the original publication, including its DOI. [DOI: 10.1117/1.JOM.2.1.011004]
\end{abstract}

Keywords: MOEMS; microscanning mirror; synchronized scanning; MEMS light detection and ranging; three-dimensional sensing; self-aligned microassembly.

Paper 21017SS received Jul. 23, 2021; accepted for publication Dec. 7, 2021; published online Jan. 8, 2022.

\section{Introduction}

Laser scanners are widely used for three-dimensional (3D) optical distance measurement systems. ${ }^{1,2,3}$ The principle of optical distance measurement is a time-of-flight $(\mathrm{ToF})$ measurement of light, also known as light detection and ranging (LiDAR), which is based on the well-defined velocity of light with $c_{0}=2.99792458 \cdot 10^{8} \mathrm{~m} / \mathrm{s}$ in vacuum. LiDAR systems measure the travelling time of light resulting from emission by a modulated light source (typically a collimated laser beam) scattering at the target, and time-resolved detection with a fast photodetector. Basis of all LiDAR techniques is the precise measurement of light travelling time $\Delta t$ to and from the measuring object, where the distance $z$ results for a measurement environment of refractive index $n$

$$
z=\frac{c_{0} \cdot \Delta t}{2 \cdot n} .
$$

\footnotetext{
*Address all correspondence to Thilo Sandner, thilo.sandner@ipms.fraunhofer.de
} 
LiDAR techniques can be divided into pulsed and phase-modulated ToF measurement techniques and frequency-modulated continuous-wave techniques. ${ }^{1}$ For a phase-modulated $\mathrm{ToF}$ technique, the light intensity of the emitter is modulated with a high frequency $f_{\text {mod }}$. The distance $z$ of the measured object results from the phase shift $\varphi$ between emitted and detected light

$$
z=\frac{c \cdot \varphi}{4 \pi f_{\text {mod }} \cdot n} \text {. }
$$

The phase $\varphi$ is only uniquely measureable within the interval $0, \ldots, 2 \pi$ (corresponds, e.g., to a distance measurement range of $1 \mathrm{~m}$ at $f_{\text {mod }}=150 \mathrm{MHz}^{2}$ ). To extend the uniqueness range of distance measurement, multiple modulation frequencies are also used. This results in a significantly smaller but higher resolution distance measurement range for a phase-modulated ToF distance measurement system (also with the advantage of simplified system complexity) in comparison with a pulsed ToF technique, which is typically used for long distance measurement ranges up to several ${ }^{2} 100 \mathrm{~m}$.

The light from the emitter is scattered at the target and requires a large aperture of the optical receiver, requiring also optical signal detection of high dynamic range, because the intensity at the detector is inversely proportional to the square of the target distance. Furthermore, pulsed ToF techniques also have the benefit that higher light intensities can be used at the target due to eye safety reasons (depending on the wavelength, where $\lambda=1550 \mathrm{~nm}$ has the advantage that light cannot damage the human retina) —advantageous to detect the laser signal from the optical background noise. A classical laser scanner combines an optical ToF distance measurement technique with a scanning mirror optic — using a synchronously scanning mirror element for both emitting and receiving optical channels-allowing light to be detected from only a small area around the actual illuminated measurement point. A 3D imaging is achieved by sequential twodimensional (2D) geometrical scanning in $x$ and $y$ combined with a ToF distance measurement in $z$. Typically, classical laser scanners are not used for real-time 3D imaging due to the limited scanning speed of conventional scanning mechanics. For real-time 3D imaging, alternative techniques exist. Flash LiDAR systems use a parallel ToF detection with 2D detector arrays and a simultaneous illumination of the entire scene. ${ }^{4,5} 3 \mathrm{D}$ ToF cameras also exist with parallel ToF detection with $2 \mathrm{D}$ array detectors [e.g. complementary metal-oxide semiconductor-singlephoton avalanche diode arrays] and nonscanned receiver optics combined with a single MEMS scanning mirror (MSM) for scanned local object illumination. ${ }^{6-8}$ These MEMS-based 3D ToF systems are promising for actual developments of LiDAR systems for autonomous driving due to their potential for cost and size reduction. ${ }^{8-10}$ Some MEMS-based LiDAR systems are also reported in Ref. 11. In Hofmann et al., ${ }^{12}$ it is reported that a resonant $550 \mathrm{~Hz}$ 2D scanner with $7 \mathrm{~mm}$ aperture is used for $360 \mathrm{deg}$ [one-dimensional (1D)] scanning of a conceptual pulsed 3D ToF automotive sensor by means of a circular scanning trajectory in combination with an omnidirectional scan lens. In Ye et al., ${ }^{13}$ a new electromagnetically driven 1D resonant MEMS mirror made of Ti-Al instead of c-Si was published for a future LiDAR application. This resonant 1D MEMS scanner has a large circular aperture of $12 \mathrm{~mm}$ and achieves an optical scan range of $26 \mathrm{deg}$ at a resonance frequency of $1240 \mathrm{~Hz}$. In Senger et al., ${ }^{14}$ a piezoelectric resonant driven 2D MEMS scanner with $20 \mathrm{~mm}$ mirror aperture was presented, resulting in a circular scan pattern at about $1445 \mathrm{~Hz}$ with only 0.1 deg optical scan range. In Schwarz et al., ${ }^{15}$ a piezoelectrically driven resonant 1D MEMS mirror with an elliptical aperture of $2 \mathrm{~mm} \times 4 \mathrm{~mm}$ and a scan frequency of about $1.5 \mathrm{kHz}$ for automotive LiDAR were presented, enabling a total optical scan angle of $180 \mathrm{deg}$. Beside LiDAR, MEMS scanners ${ }^{16}$ are also interesting for further 2D/3D measuring techniques that require fast optical scanning, e.g., for high-speed imaging of unsteady spray flows using frequency-modulated Doppler global velocimetry. ${ }^{17}$

In spite of the alternative 3D ToF techniques, classical laser scanners have the advantage of higher measurement accuracy (e.g., in comparison with focal plane array-based 3D ToF cameras) due to the synchronous scanning principle, because only single measuring points within the scanned field of view (FOV) are sequentially illuminated. Hence, the LiDAR detector collects light from signal and background (noise) in a short time only from a small measured area of the target, which significantly reduces the influence of the background (noise). Also, a nearly unlimited number of 3D data (voxels) can be measured with high measuring rates of typically up to 
$1 \times 10^{6} \mathrm{voxel} / \mathrm{s}$ and low measurement uncertainty. On the other hand, conventional laser scanners for 3D distance measurement involve expensive, heavy, and large rotating or vibrating mirrors for light deflection of the scanning ToF distance measurement. ${ }^{2}$ Typically, the precision of the ToF distance measurement is limited by the amount of signal light available at the detector. Hence, a scanning mirror with a large aperture is required for the optical receiver of the LiDAR system to detect small amounts of light reflected or scattered by the measured target. Traditionally, polygon scanners are used, but for high scanning speeds (e.g., $>30,000 \mathrm{rpm}$ ) great efforts (e.g., air bearings) are required to reduce abrasive wear, resulting in heavy (>10 kg) and costly scanning units. Replacing them with micromechanical scanning mirrors, which have the benefits of high scanning speed, low weight, high mechanical reliability (e.g., no friction and high shock resistance), is not straightforward, since a large mirror aperture of the receiver optics must also be guaranteed in addition to sufficiently large optical scan angles ( $>40 \mathrm{deg}$ ) and high scan frequencies of more than $100 \mathrm{~Hz}$. Contrary to this, the aperture of a single MSM is limited to small values of typically $1, \ldots, 5 \mathrm{~mm}$ diameter due to dynamic mirror deformation. ${ }^{16,18}$

\subsection{MEMS-Based ToF LaserScanner}

To overcome the aforementioned problems of a classical laser scanner, Fraunhofer developed the concept for a MEMS-based LiDAR laser scanner in $2008,{ }^{19}$ which uses an array of identical MEMS elements driven in a synchronized mode. This concept was motivated for industrial high-speed 3D LiDAR systems used, e.g., in railroad inspection. A first prototype LAMDA (i.e., Large Aperture MEMS scanner module for 3D Data Acquisition) ${ }^{20}$-developed for a phase-shifting distance measuring system-was limited to resonant 1D scanning at $250 \mathrm{~Hz}$ and $60 \mathrm{deg}$ FOV using a monolithic MSM array with $80 \%$ optical filling factor and a total aperture of $334.2 \mathrm{~mm}^{2}$. It consists of $2 \times 7$ MEMS mirrors driven synchronized to a separate sending mirror [see Fig. 1(b)]. For mirror synchronization, the LAMDA system uses hybrid integrated optical position detectors. ${ }^{20}$

In Sandner et al., ${ }^{21}$ this MEMS-based LiDAR was extended by the concept of the adaptive 3D ToF laser camera TACO (i.e., Three-dimensional Adaptive Camera with Object detection and foveation) with foveation properties (see Fig. 2), e.g., to allow future autonomous robots to better interact with their environment. This 3D sensor concept of foveation-i.e., capturing distance images at video-like frame rates with coarse spatial resolution, rapidly detecting regions of interest (ROIs), and then concentrating further image acquisition on these ROIs using adaptive scanning - required a sophisticated 2D scanning device with quasi-static actuation, large effective aperture $\geq 5 \mathrm{~mm}$, high scanning rates $>1000 \mathrm{~Hz}$, and a large optical scan range of FOV $\geq 60 \mathrm{deg}$.

For the TACO sensor, ${ }^{21}$ the best technical compromise for building a fast adaptive scanning unit was found in a quasi-static/resonant raster-scanning MEMS mirror. Using vertical out-of-
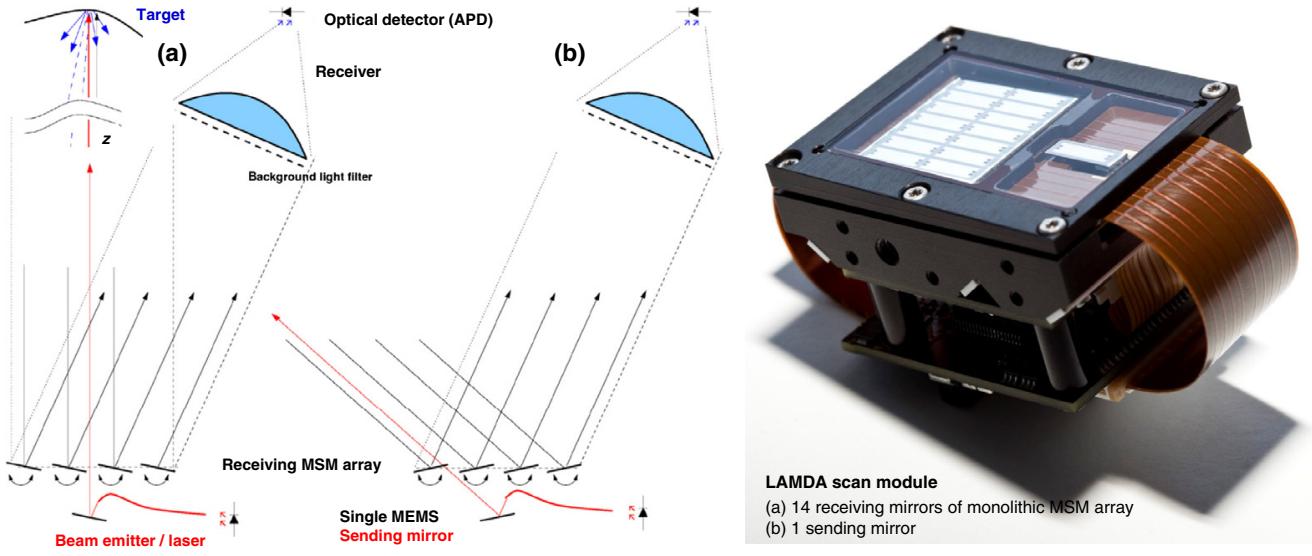

Fig. 1 Basic concept of MEMS-based LiDAR using MEMS scanner arrays for receiver channel (left); large aperture MEMS scan module LAMDA with monolithic 1D MSM array with $250 \mathrm{~Hz}$ resonance frequency and $80 \%$ optical filling factor and separate sending mirror ${ }^{20}$ (right). 
(a)

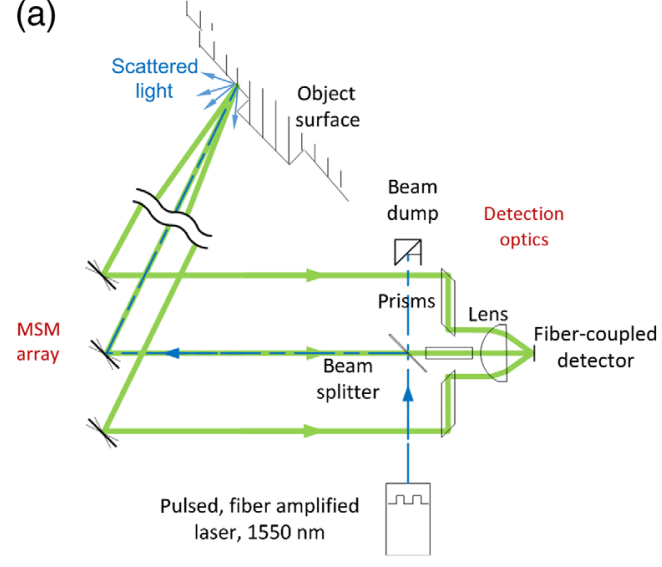

(b)
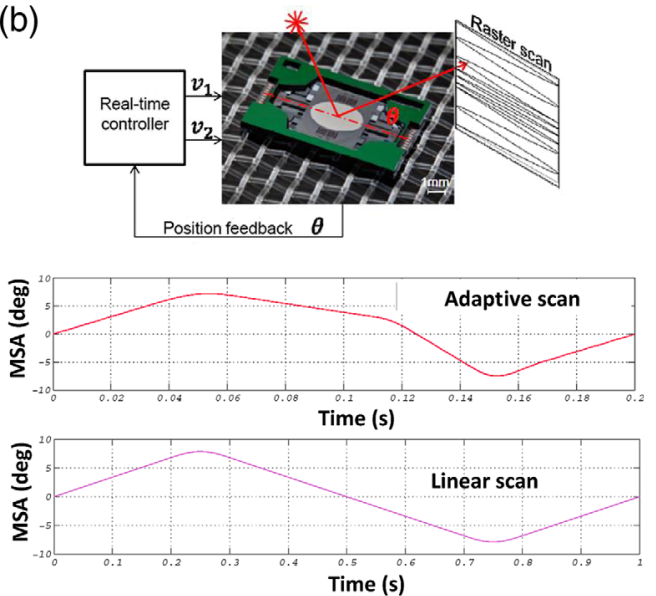

Fig. 2 Concept of adaptive MEMS-based 3D laser camera TACO ${ }^{21}$ : schematic optical setup using a synchronized $2 \mathrm{D}$ raster MSM array to provide sufficient reception aperture (a) and 2D raster scanning mirror with adaptive vertical scanning enabling partial foveation with higher resolution in ROls (b).

plane comb drives—based on the quasi-static LinScan driving principle ${ }^{22,23}$ —a quasi-static mechanical scan angle (MSA) of $\pm 10 \mathrm{deg}$ as well as a linearized or adaptive scanning ${ }^{24,25}$ with partial foveation in the vertical direction was realized, enabling also a variable frame rate of $1, \ldots, 100 \mathrm{~Hz}(10 \mathrm{~Hz}$ typically). The TACO system uses a synchronized MSM array consisting of five hybrid assembled and synchronously driven 2D MEMS mirrors-selected by frequency of the fast horizontal scan axis with $3200 \mathrm{~Hz}$ bidirectional scanning rate. The TACO system uses a fiber amplified pulsed ToF distance measurement system at $\lambda=1550 \mathrm{~nm}$ with 1 MVoxel 3D measurement rate and $5 \mathrm{~mm}$ distance uncertainty at $7.5 \mathrm{~m}$ measuring range.

For TACO, the hybrid MEMS array is built in a symmetric coaxial assembly of four peripheral receiving mirrors around the centered sending mirror, ${ }^{21,26}$ see Fig. 3(a), to provide the effective reception aperture of $5 \mathrm{~mm}$. Due to the limited optical filling factor $<20 \%$ of the 2D MSM, optical guiding prisms are used to transfer the light collected by the peripheral MEMS mirrors near to the optical axis. This setup of the optical receiver channel enables focusing of the received light (backscattered from the target) onto the fiber-coupled avalanche photodiode detector with a fiber core diameter of only $100 \mu \mathrm{m}$ [see Fig. 3(a)]. Details of the optical sensor design are found in Ref. 26.

This paper focuses on a fast MSM array with large aperture developed for the 3D laser scanner Fovea3D (i.e., adaptive 3D sensing with foveation) with panoramic FOV (60 deg $\times$ $360 \mathrm{deg}$ ). This paper is an extended work of Ref. 27, where we publish now several details of the MEMS system integration for the first time, e.g., (i) a self-aligned microassembly technique of the hybrid MSM array using wet-etched pyramidal self-alignment structures, and (ii) self-compensation structures for reducing deep reactive-ion etching (DRIE) caused tolerances of the resonance frequency. In Sec. 2, the concept of the 3D ToF camera Fovea3D is introduced. Section 3 presents the MEMS design and fabrication. In Sec. 4, experimental results of single MEMS scanner elements are shown. The system integration of the hybrid assembled MEMS scanner array is discussed in Sec. 5. Finally, this paper is concluded in Sec. 6, and an outlook on future applications of the presented MEMS technology is given in Sec. 7.

\section{Concept of the 3D TOF Camera Fovea3D}

In Sandner et al. ${ }^{27}$ we extend the basic concept of the MEMS-based adaptive 3D ToF laser camera with foveation properties to longer measuring distances. It targets on a measuring range of up to $100 \mathrm{~m}$, needed for outdoor security applications (e.g., observation of industrial objects, airports, etc.). The 3D ToF camera Fovea3D is based on the same pulsed ToF laser distance measurement system—developed for the TACO sensor ${ }^{21}$ —and also uses a pulsed fiber amplified 
Table 1 Target specification of the 3D camera Fovea3D in comparison with the TACO system.

\begin{tabular}{lccc}
\hline \hline Parameter & Units & Fovea3D & TACO $^{21}$ \\
\hline Distance min & $\mathrm{cm}$ & 10 & 10 \\
Distance max & $\mathrm{m}$ & $100(30$ typ. & 10 (7.5 typ.) \\
FOV horizontal & $\mathrm{deg}$ & 120 oscillating (360 rotating) & 60 (80 max) \\
FOV vertical & $\mathrm{deg}$ & 60 & 40 \\
Beam divergence & $\mathrm{mrad}$ & 2 at $\lambda=1550 \mathrm{~nm}$ & 2 at $\lambda=1550 \mathrm{~nm}$ \\
3D measuring rate & Voxel/s & $10^{6}$ & $10^{6}$ \\
Distance accuracy & $\mathrm{mm}$ & 6 at $30 \mathrm{~m}$ & 5 at $7.5 \mathrm{~m}$ \\
Frame rate; variable & frame/s & DC, $1, \ldots, 10$ & $1, \ldots, 100 ; 25$ at $240 \times 160$ pixels \\
Fast scan rate (bidirectional) & $\mathrm{Hz}$ & 3200 (vertical) & 3200 (horizontal) \\
Foveation (adaptive scanning) & Direction & Horizontal & Vertical \\
\hline \hline
\end{tabular}

laser system with $\lambda=1550 \mathrm{~nm}, P_{\text {pulse }}=1.5 \mathrm{~kW}, P_{\text {average }}=1.5 \mathrm{~W}$, and 1 MVoxel measuring rate. To enable an enlarged distance measuring range of $100 \mathrm{~m}$, the reception aperture had to be significantly increased to an effective aperture of $D_{\text {eff }}=23 \mathrm{~mm}$ in comparison with only $D_{\text {eff }}=$ $5 \mathrm{~mm}$ used for the TACO sensor (with a limited distance measuring range of only $10 \mathrm{~m}$ ). The target specifications of the 3D camera Fovea3D are summarized in Table 1 in comparison with the previous TACO system.

Table 1 shows the following improvements for the Fovea3D system:

1. larger effective aperture $D_{\text {eff }}=23 \mathrm{~mm}$,

2. vertical optical scan range $\mathrm{FOV}=60 \mathrm{deg}$,

3. horizontal optical scan range $\mathrm{FOV}=360 \mathrm{deg}$ (rotational),

4. rotational frequency up to $600 \mathrm{rpm}(10 \mathrm{~Hz}$ frame rate) with horizontal foveation.

The Fovea3D system should also acquire 3D distance images at video-like frame rates with coarse spatial resolution, rapidly detecting ROIs and then concentrating further image acquisition on these ROIs with adaptive scanning in the horizontal direction. From the optical setup of the previous TACO system (see Fig. 3), it is obvious that the 2D MEMS approach is not scalable to meet the specifications required for the Fovea3D system. This holds particularly true for the enlarged aperture of $D_{\text {eff }}=23 \mathrm{~mm}$.

To enable the challenging specification of the 3D camera Fovea3D, the following system concept was used (see Fig. 4):

(a)

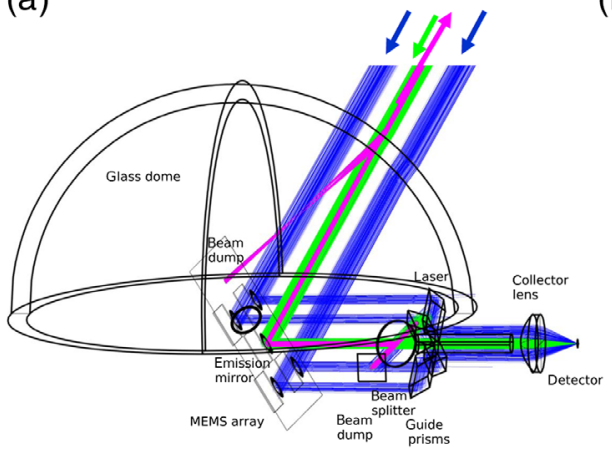

(b)

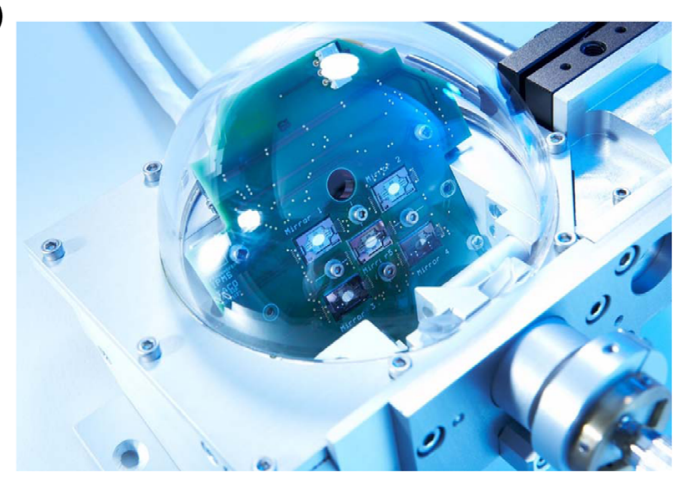

Fig. 3 Adaptive 3D ToF camera TACO ${ }^{21}$ : (a) schematic setup of optical scan head ${ }^{26}$ and (b) scan head with hybrid assembled 2D MEMS raster scanner array. 
(a)

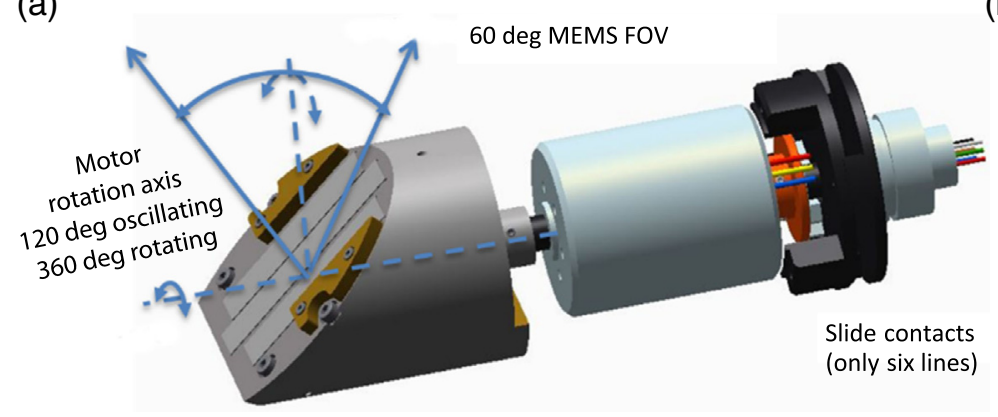

(b)

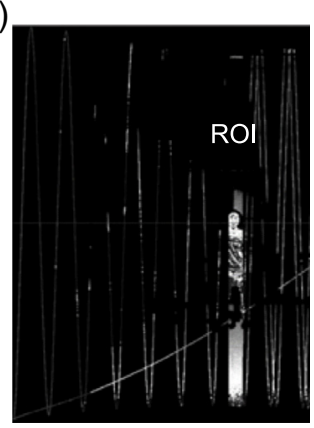

Fig. 4 (a) 3D computer-aided design (CAD) model of the Fovea3D scan head and (b) principle of foveation by adaptive horizontal scanning.

1. use of a conventional electro-mechanical drive for horizontal scanning:

a. enabling linear or adaptive scanning with 360 deg rotation or 120 deg oscillation,

2. resonant 1D MEMS array with increased optical aperture and larger filling factor with a simplified optical setup,

3. fast resonant MEMS scanning in vertical direction enabling:

a. reduced number of scan elements until $D_{\text {eff }}=23 \mathrm{~mm}$,

b. reduced form factor and complexity of MEMS scan module,

c. larger vertical FOV of $60 \mathrm{deg}$,

d. enlarged frequency bandwidth of MEMS scanner reducing the risks for synchronization of multiple MEMS scanner elements.

In contrast to the monolithic $1 \mathrm{D}$ MSM array LAMDA, ${ }^{20}$ the 1D MEMS scanner array (developed for Fovea3D) was realized by hybrid assembly of frequency selected single scanner elements [see Fig. 5(a)]. The Fovea MSM array consists of 22 receiver scanner elements (optimized for a large single aperture of $2.4 \times 8.4 \mathrm{~mm}^{2}$ ). They are hybrid assembled around the coaxial sending mirror optimized for reduced dynamic mirror deformation and low beam divergence of $1 \mathrm{mrad}$ at $\lambda=1500 \mathrm{~nm}$. The hybrid assembly of fast scanning 1D MEMS array (using a self-alignment microassembly technique) was chosen because of the small frequency bandwidth $\leq 10 \mathrm{~Hz}$ available for synchronized operation of all individual scanner elements. The approach of hybrid assembled MEMS array enables:

1. increased MEMS yield and reduced overall costs using frequency selected scanner chips,

2. simplified rework of a damaged scan element.

(a)

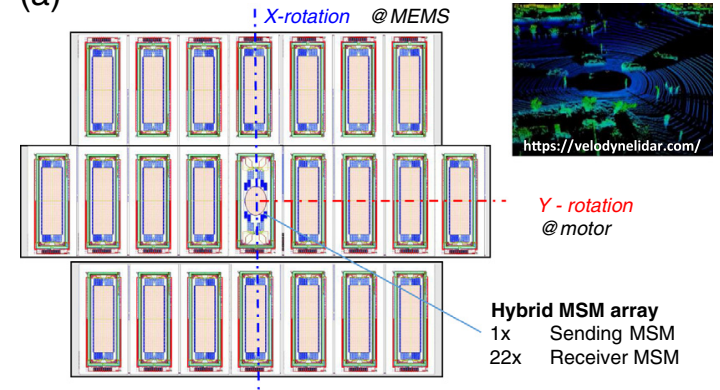

(b)

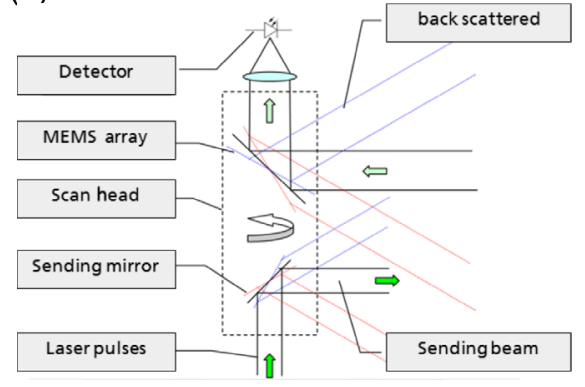

Fig. 5 System concept of the adaptive 3D laser camera Fovea3D: (a) schematic setup of the hybrid assembled 1D MSM array (developed by Fraunhofer IPMS) for fast vertical scanning with $3200 \mathrm{~Hz}$ bidirectional scan rate and (b) optomechanical concept of the scan head using 1D MSM array for fast vertical and a conventional electromagnetic drive for horizontal scanning. 
To provide the effective reception aperture of $23 \mathrm{~mm}$, all receiving MEMS mirrors are precisely synchronized in respect to the oscillating sending mirror (see Fig. 5). In comparison with state-ofthe-art 360-deg laser scanners of the Velodyne company, e.g., the panoramic 3D camera HDL$64 \mathrm{E},{ }^{28}$ which uses a rotational motor combined with 64 parallel and multiplexed single-channel ToF detectors over 26.9 deg vertical FOV, the Fovea3D camera can provide a larger vertical FOV of 60 deg with significantly increased vertical angular resolution due to MEMS scanning (limited only by the $1 \mathrm{mrad}$ optical divergence of the collimated pulse laser beam).

\section{MEMS Design and Fabrication}

The resonant 1D MEMS scanners presented in this paper were especially designed for the system concept of the adaptive 3D ToF camera Fovea3D (shown in Figs. 4 and 5). Two separate MEMS scanner designs were developed and optimized for the use as sending and receiving mirrors. The MEMS design targets on the following items:

1. enhancement of frequency bandwidth of a single scanning mirror to guarantee robust synchronization of the MSM array,

2. enlargement of single receiver aperture $(2.4 \mathrm{~mm} \times 8.4 \mathrm{~mm})$ to reduce the number of receiver elements required for full receiver aperture of $D_{\text {eff }}=23 \mathrm{~mm}$,

3. guarantee a low beam divergence of $1 \mathrm{mrad}$ and minimization of dynamic mirror deformation of sending mirror $\leq 155 \mathrm{~nm}(\leq \lambda / 10$ at $\lambda=1550 \mathrm{~nm})$.

Both scanning mirror designs were based on the electrostatic resonant driving principle of Fraunhofer Institute for Photonic Microsystems (IPMS). ${ }^{18}$ Typically, these resonant microscanning mirrors are driven in parametric resonance. ${ }^{29}$ For excitation in parametric resonance, a pulsed driving voltage $U_{\text {Drive }}$ with $f_{\text {pulse }}=2 \cdot f_{\text {oscillation }}$ pulse driving frequency and $50 \%$ duty cycle are used. A MEMS oscillation occurs only in a limited parameter range of $U_{\text {Drive }}$ and $f_{\text {pulse }}$, the so called electrostatic stability region, where the maximal usable driving voltage is limited by the pull-in voltage $U_{\text {pull-in }}$ of the MSM. Typically, the resonant MEMS scanner starts with a frequency down-sweep using a fixed driving voltage. The MEMS oscillation occurs when the limit of the stability region of the parametric resonance regime is exceeded. The correlation between electrostatic stability region and voltage-dependent amplitude-frequency characteristics is exemplarily shown in Fig. 6 for the resonant $1600 \mathrm{~Hz}$ inner axis of the 2D MEMS scanner used in the TACO system (shown in Fig. 2), which is the reference for the Fovea3D design.

The specifications of sending and receiving mirrors are summarized in Table 2.

In Fig. 7, the finite element analysis (FEA) models of both 1D MEMS scanner designs are exemplarily shown for the first eigenmode (used for scanning). The FEA simulation results of the dynamic mirror deformation are shown in Fig. 8 for nominal deflection at MSA $= \pm 15 \mathrm{deg}$ and $1600 \mathrm{~Hz}$. For the sending mirror, a distributed spring suspension ${ }^{18}$ of the elliptic $3.3 \mathrm{~mm} \times$ $3.6 \mathrm{~mm}$ mirror plate was used to minimize the dynamic deformation to $\delta_{\mathrm{PP}}=106.0 \mathrm{~nm}<\lambda / 10$

(a)

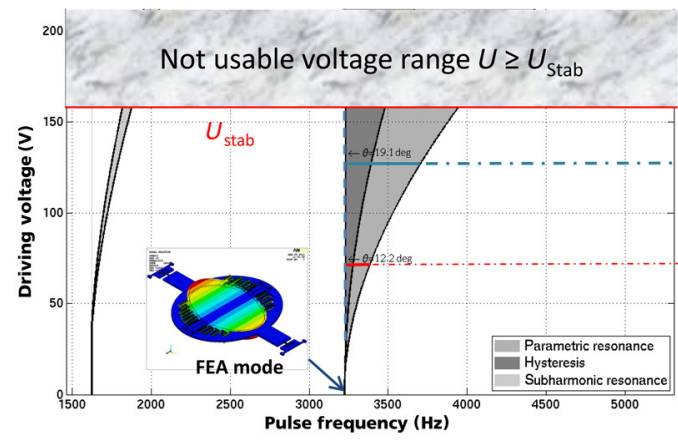

(b)

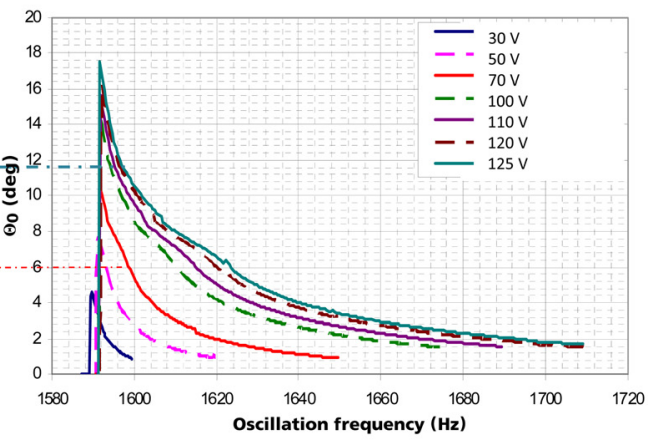

Fig. 6 (a) Correlation of electrostatic stability region of parametric resonant driving and (b) amplitude-frequency response curves. 
Sandner et al.: System integration of hybrid assembled large aperture Micro Scanner Array...

Table 2 Specification of 1D MEMS scanner designs developed for the Fovea3D sensor.

\begin{tabular}{lccc}
\hline \hline Parameter & Units & Sending mirror & Receiving mirror \\
\hline Mirror aperture & $\mathrm{mm}$ & $3.3 \times 3.6$ & $2.4 \times 8.4$ \\
Dynamic deformation (root mean square) & $\mathrm{nm}$ & 25 at $15 \mathrm{deg}$ & 975 at $15 \mathrm{deg}$ \\
Eigen frequency & $\mathrm{Hz}$ & 1610 & 1599 \\
Nominal mechanical scan angle $\mathrm{MSA}_{\text {nom }}$ & $\mathrm{deg}$ & 15 & 15 \\
Maximal mechanical scan angle $\mathrm{MSA}_{\max }$ & $\mathrm{deg}$ & 20 & 20 \\
$U_{\text {drive }}$ at MSA & $\mathrm{V}$ & 180 & 165 \\
Frequency bandwidth BW at $15 \mathrm{deg}$ & $\mathrm{Hz}$ & 16.3 at $240 \mathrm{~V}$ & 11.6 at $250 \mathrm{~V}$ \\
Pull-in voltage $U_{\text {pull-in }}$ & $\mathrm{V}$ & 250 & 257 \\
Chip size & $\mathrm{mm}$ & $13.81 \times 6.39$ & $13.81 \times 5.91$ \\
\hline \hline
\end{tabular}

(a)

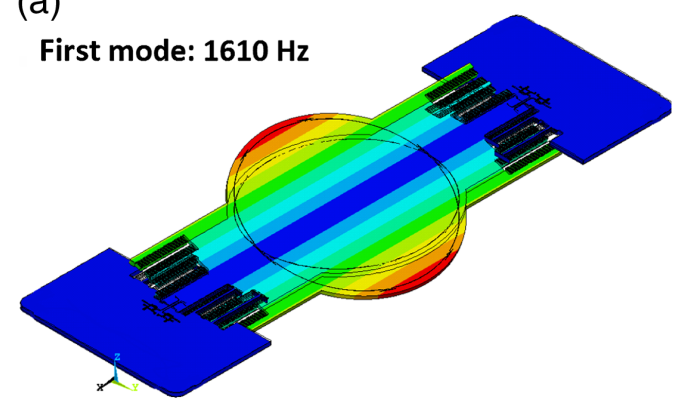

(b)

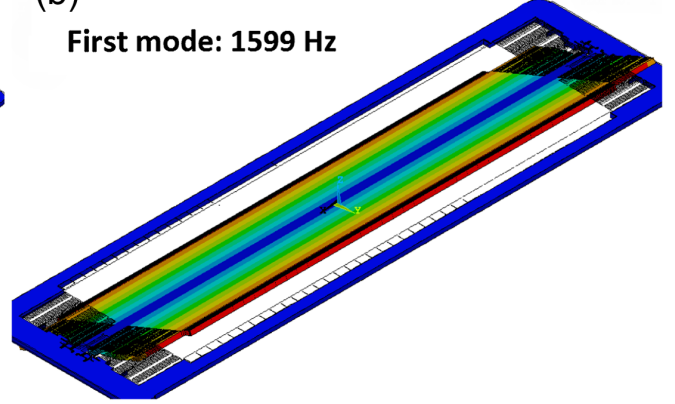

Fig. 7 FEA model of 1D MSM: (a) sending mirror and (b) receiving mirror.

(a)

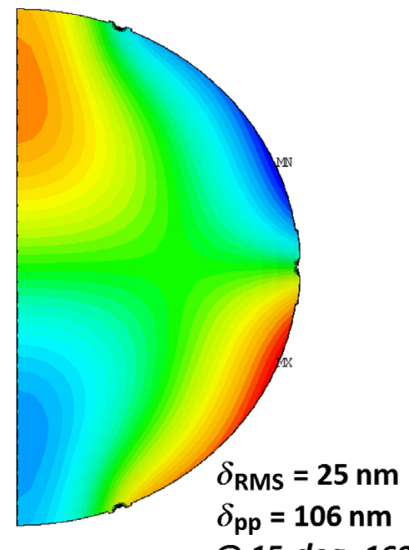

(b)

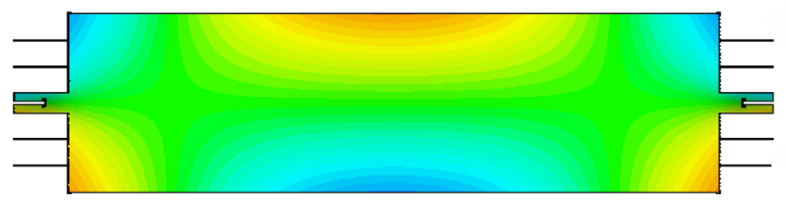

RMS = 975 nm @ $15 \mathrm{deg} ; 1600 \mathrm{~Hz}$

Fig. 8 FEA results of dynamic mirror deformation at \pm 15 deg and $1600 \mathrm{~Hz}$ : (a) sending MSM and (b) reception MSM.

and $\delta_{\mathrm{RMS}}=25.0 \mathrm{~nm}$, respectively, enabling high optical resolution of the scanned laser spot at the target. For the receiver MSM, a larger deformation exists, which influences the coupling efficiency of the fiber-coupled photodetector only slightly.

In Fig. 9, the electrostatic stability region (i.e., the parameter range of the MEMS driving in parametric resonance) is exemplarily shown for the first, second, and third eigenmodes of the sending MEMS mirror as well as the first and third subharmonic modes. The tilting mode used is 


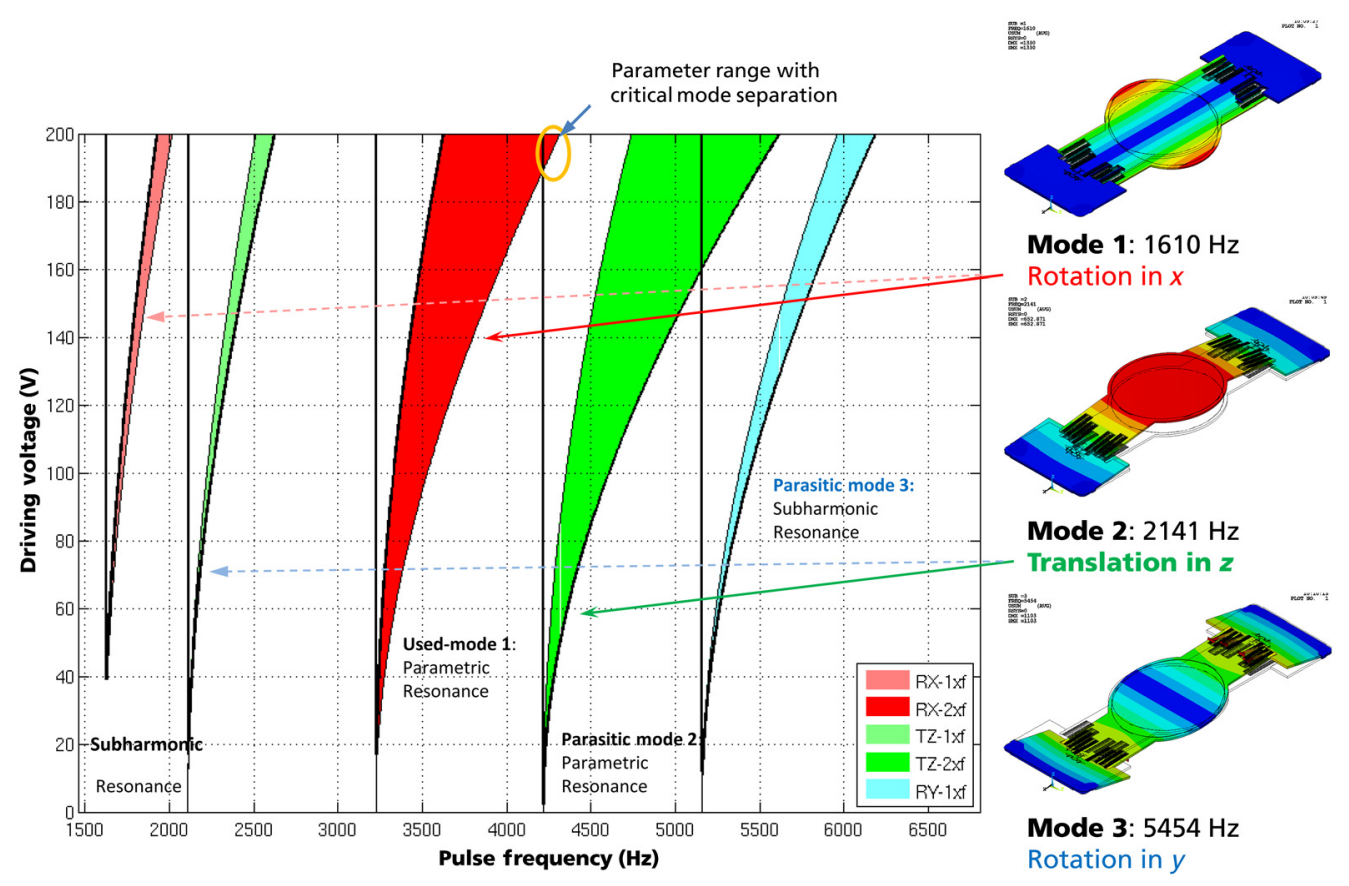

Fig. 9 Stability regions of electrostatic actuation in parametric resonance, shown for first, second, and third eigenmode of sending MSM.

the first mode $f_{1}=1610 \mathrm{~Hz}$. For precise rotational MEMS oscillation, any overlapping of the electrostatic stability regions of the first mode used with any parasitic or subharmonic modes has to be avoided by the MEMS design within the practically relevant range of driving parameters. In Fig. 9, a good mode separation is obvious for the sending mirror for all relevant driving voltages $\leq 180 \mathrm{~V}$. Hence, a precise MEMS rotation in $x$ (for the sending mirror shown) is guaranteed by the MEMS design.

For MEMS fabrication, bonded silicon-on-insulator (BSOI) substrates with highly $p$-doped 75- $\mu \mathrm{m}$ thick device (SOI) layers were utilized. Details of the used qualified bulk micromachining MEMS scanner process (AME75) are found in Ref. 18. In contrast to the previous 1D MSM array LAMDA, ${ }^{20}$ we included monolithically integrated piezoresistive (PZR) position sensors ${ }^{30}$ for the design of the Fovea scanner to simplify the efforts for MEMS system integration. Photographs of the fabricated MSMs are shown in Fig. 10.

Details of the reception MSM are shown in Fig. 11. A challenge for the MEMS manufacturing was to ensure low tolerances of the resonant frequency due to the small frequency bandwidth $<8 \mathrm{~Hz}$ at $U_{\max }=180 \mathrm{~V}$ available for synchronous driving of multiple MEMS elements at nominal deflection (MSA $=15 \mathrm{deg}$ ). To reduce the DRIE fabrication tolerances on the resonance frequency, we use latticed self-compensation structures located on the outer edge of the mirror plate [see Figs. 11(b) and 11(c)]. The self-compensation effect on the resonance frequency is enabled by reducing the moment of mirror inertia (by means of the latticed structures) in case the DRIE-etched trenches become too wide (resulting in a reduced spring constant and lower

(a)

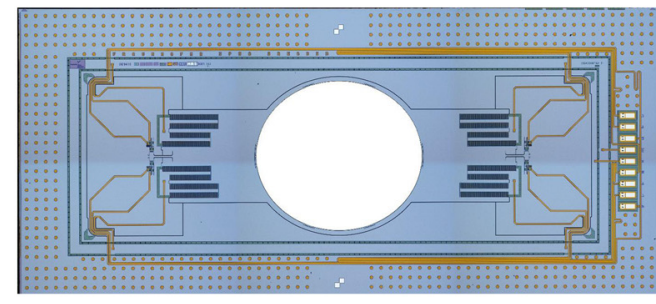

(b)

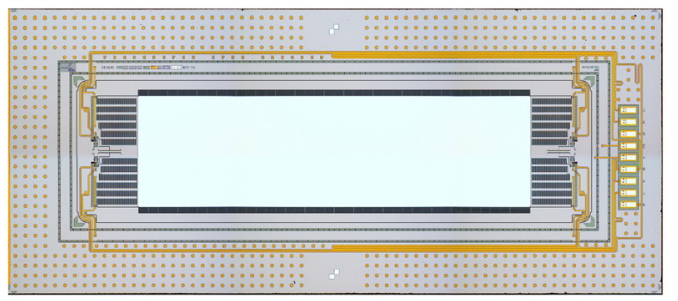

Fig. 10 Photographs of the MEMS scanner chip: (a) sending MSM and (b) reception MSM. 
(a)

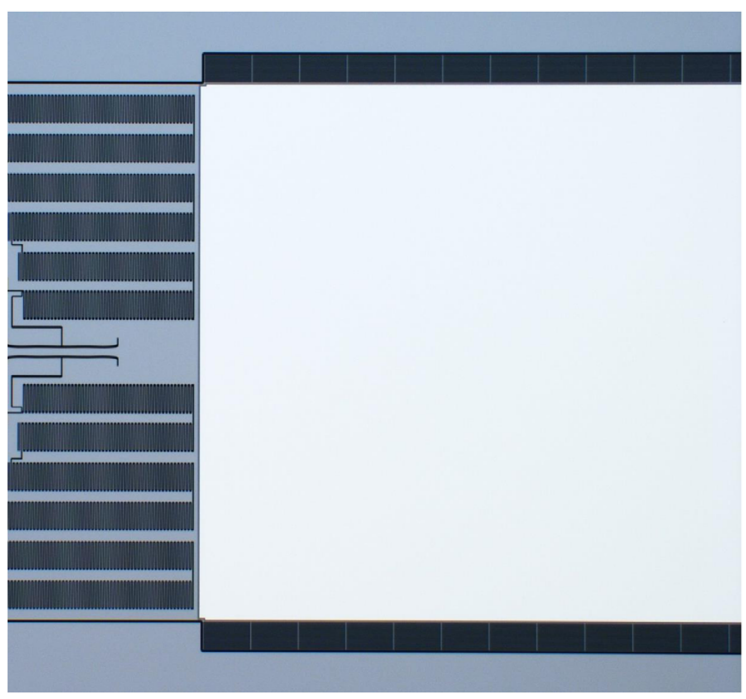

(b)

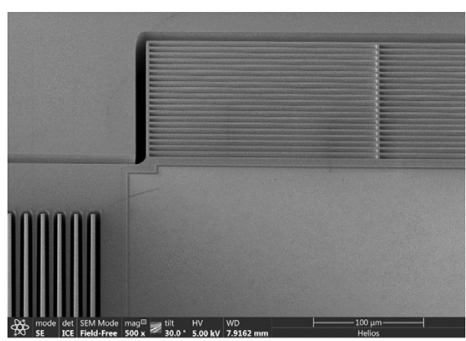

(c)

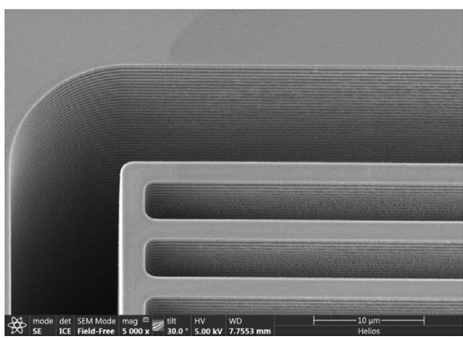

Fig. 11 (a) Photographic details of reception MSM. (b) and (c) scanning electron microscope (SEM) details of the compensation structures for reducing DRIE caused frequency tolerances.

resonance frequency, respectively). In the opposite case of DRIE-etched trenches that are too narrow, the spring constants increases, but the increases in resonance frequencies are selfcompensated by the increased moment of mirror inertia caused by the latticed compensation structures.

\section{Experimental Results}

The voltage-dependent amplitude frequency response characteristics of a receiving mirror are exemplarily shown in Fig. 12. They were measured in parametric resonance for varied pulse voltages (up to $180 \mathrm{~V}$ ) and $50 \%$ duty cycle using an optical deflectometry setup with discrete photodiodes to measure the scan amplitude. A significantly wider frequency bandwidth of $7.5 \mathrm{~Hz}$ at $\mathrm{MSA}=15 \mathrm{deg}$ is obvious for the new Fovea receiver mirror compared with the

(a)
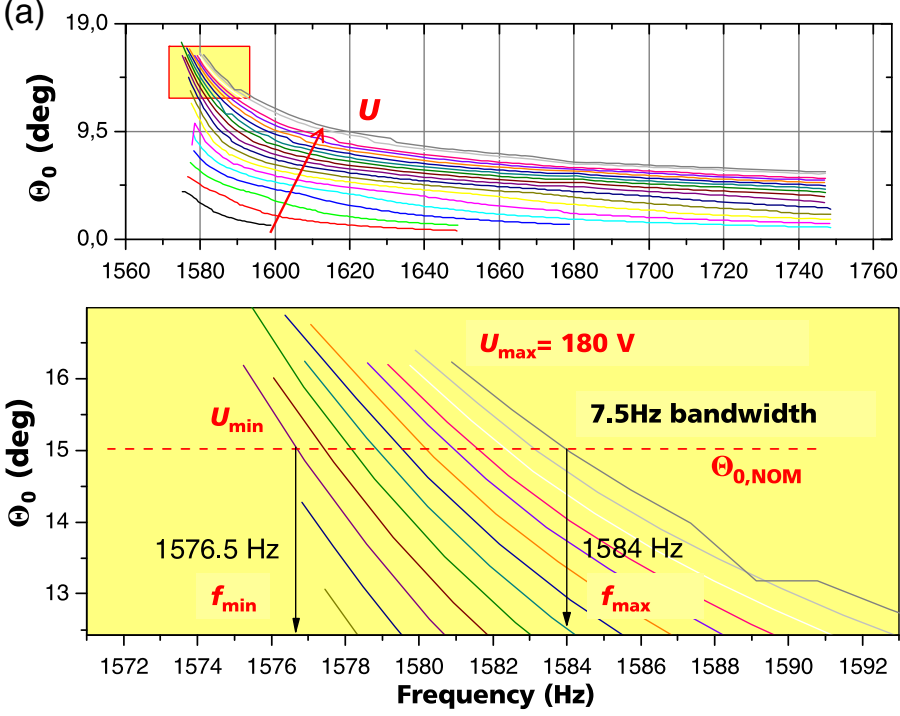

(b)

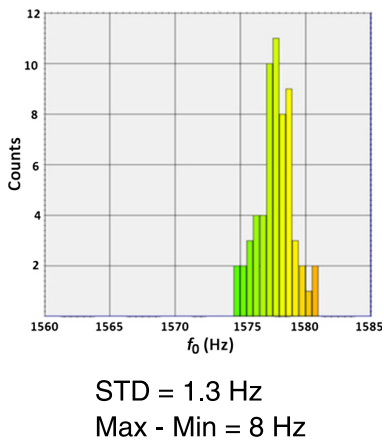

Fig. 12 (a) Amplitude-frequency response curves of receiving MEMS mirrors parameterized by the pulse driving voltage and (b) statistical distribution of the eigenfrequency of the receiver MSM of a single 6" wafer. 


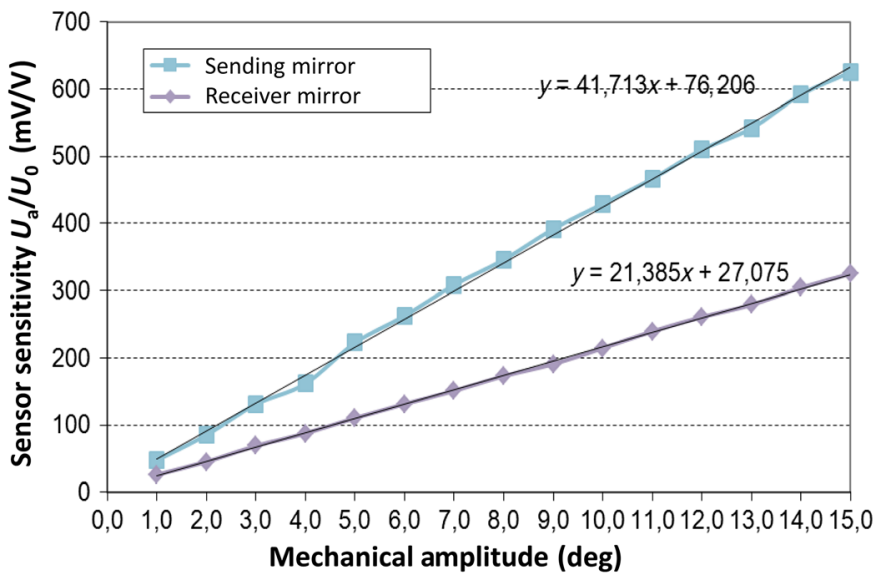

Fig. 13 Sensor characteristics of PZR position sensors.

previous TACO design ${ }^{21}$ (having a limited bandwidth of only $1.3 \mathrm{~Hz}$ at MSA $=15.2 \mathrm{deg}$, see Fig. 6).

The increased frequency bandwidth of $7.5 \mathrm{~Hz}$ of the Fovea3D scanner now enables sufficient margin for adequate MEMS yield and robustness to environmentally influenced changes in resonance frequency (e.g., temperature and ambient pressure). By means of the latticed selfcompensation structures, lower frequency tolerances were achieved. The experimental data of the statistical distribution of the eigenfrequency are shown in Fig. 12(b) (measured for a single 6" wafer). A standard deviation of STD $=3.8 \mathrm{~Hz}$ was measured for a batch of five 6" wafers. The STD is smaller than the frequency bandwidth of $7.5 \mathrm{~Hz}$. Hence, a small monolithic MSM array with increased optical fill factor (e.g., consisting of $1 \times 3$ or $2 \times 3$ scanner elements) could in principle be realized without reducing yield. However, this was out of the scope of this project.

For prospective real-time feedback control, PZR position sensors were integrated on the chip designed as a full Wheatstone bridge. ${ }^{30}$ The PZR sensor characteristics are shown in Fig. 13. A sufficient sensitivity of $S=320 \mathrm{mV} / \mathrm{V}(620 \mathrm{mV} / \mathrm{V})$ was measured for the receiver (sending) mirror design at a nominal deflection of MSA $=15 \mathrm{deg}$. A linearity error of the PZR sensors $<1 \%$ was measured for the full scanning range of MSA $=0, \ldots, 15 \mathrm{deg}$ and $<0.1 \%$ for the reduced range of MSA $=0, \ldots, 13 \mathrm{deg}$.

\section{System Integration of the MEMS Scanner Array}

\subsection{Self-Aligned Microassembly of MEMS Scanner Array}

To enable synchronized operation of multiple MEMS elements with reasonable MEMS yield and costs, a hybrid assembly was chosen (for risk management reasons) to build up the hybrid MSM array instead of the monolithic MSM array originally used in Ref. 20. Therefore 23 frequency-selected MSM elements (required for the full reception aperture of $D_{\text {eff }}=23 \mathrm{~mm}$ ) had to be precisely mounted using a newly developed microassembly technique with self-alignment. The geometrical self-alignment of the MEMS scanners was achieved by (i) a base plate with pyramidal MESA structures (see Fig. 14) and (ii) corresponding inverse pyramidal cavities etched inside the MEMS frame (see Fig. 15).

The pyramidal alignment structures were well defined by anisotropic wet-chemical etching of $\langle 100\rangle$ oriented monocrystalline silicon using tetramethylammonium hydroxide (TMAH). The pyramidal alignment structures were arranged in orthogonal lines to precisely define the horizontal and vertical MEMS positions (see Fig. 15). The pyramidal MESA structures were deliberately over-etched at the convex corners to obtain a complete resolution of the etching compensation structures to guarantee a precise form-fit connection of MESA structure and MEMS scanner.

The silicon base plate (consisting of two separate parts) was glued to an aluminum base plate with epoxy (see Fig. 17). Next, the MEMS scanner elements were manually placed and bonded 
(a)

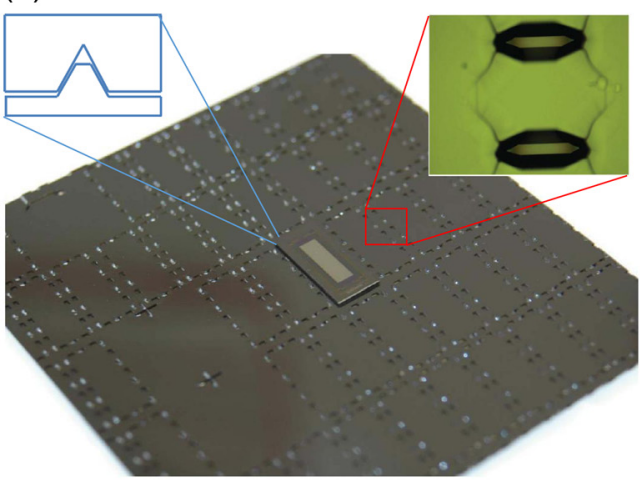

(b)

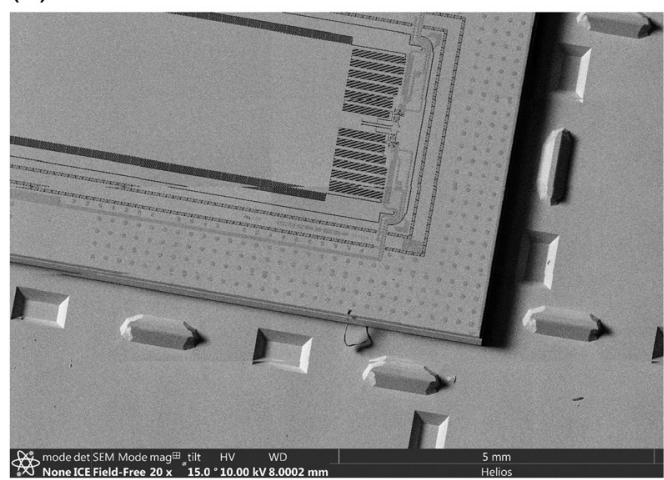

Fig. 14 Concept for self-aligned microassembly of hybrid MSM array: (a) silicon base plate with pyramidal MESA structures for geometrical self-alignment of MEMS scanner position and (b) SEM detail of self-aligned MEMS chip.

(a)

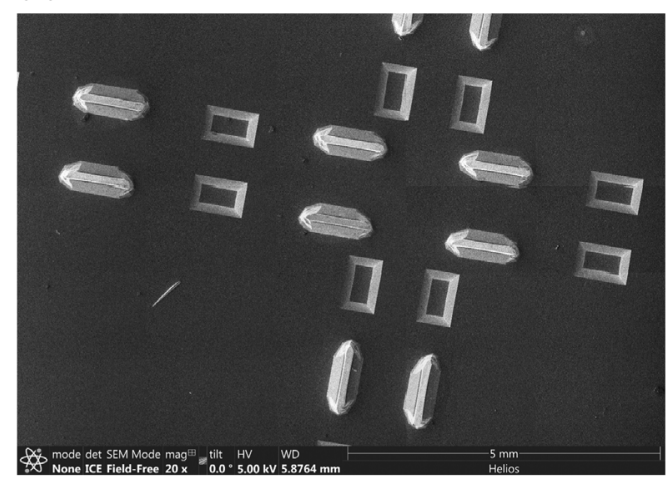

(b)

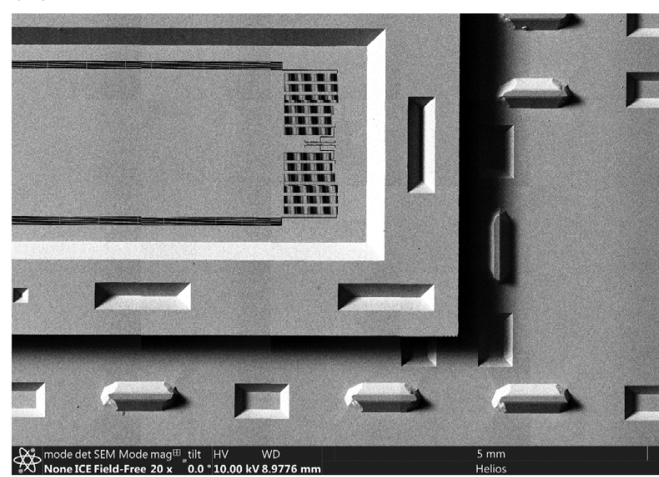

Fig. 15 SEM details of self-aligned microassembly: (a) TMAH-etched pyramidal alignment structures for horizontal and vertical scanner position and (b) backside of MEMS scanner with TMAHetched pyramidal cavities.

(a)

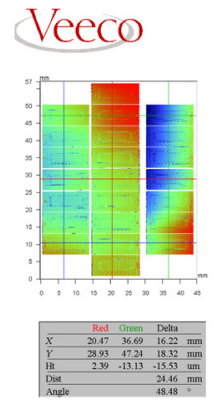

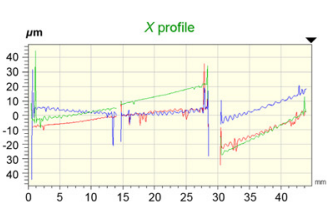
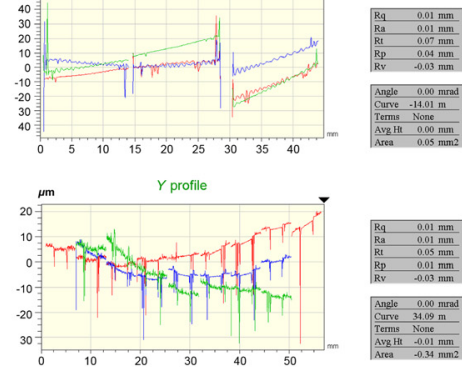

(b)

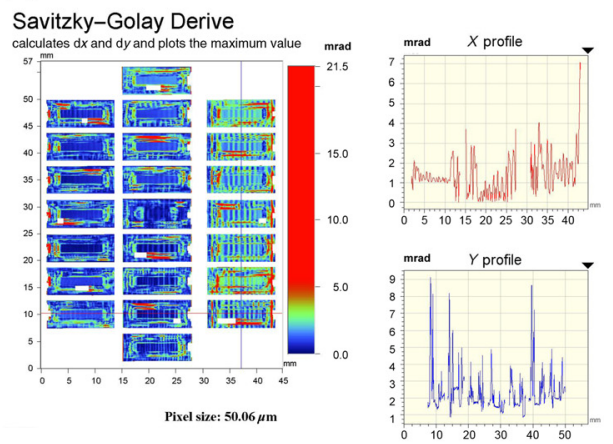

Fig. 16 Experimental results of the hybrid microassembled MSM array: (a) 3D topology of MSM array measured with WLI and (b) angular tolerances of microassembled MSM array are $<2 \mathrm{mrad}$.

to the silicon base plate with epoxy resin. The 3D topology of the microassembled MSM arraymeasured with a white light interferometer (WLI) - is shown in Fig. 16. Although only a manual assembly was used, the hybrid assembled MSM array achieved small angular tolerances $<2 \mathrm{mrad}$ (within the optically active apertures) and small lateral tolerances $<10 \mu \mathrm{m}$ of the individual MEMS scanner elements with respect to each other. The resulting range of angular tilt of 
the individual scanners of $\sim 2 \mathrm{mrad}$ (optical $0.12 \mathrm{deg}$ ) is mainly caused by the mounting accuracy of the silicon base plate and the flatness of the aluminum support structure. The self-aligned microassembly on the MESA structures, on the other hand, did not show any significant gain in the spread of the presteering of the scan angles.

\subsection{MEMS Scanning Head Fovea3D}

Details of the optical MEMS scan head, including the MEMS driver electronics and hybrid MEMS scanner array, are shown in Fig. 17 (see also Refs. 27 and 31). Field-programmable gate array (FPGA)-based MEMS driving electronics were developed by IPMS for precise synchronized operation of all individual receiver MSM elements with respect to the sending mirror of the ToF laser scanner. The entire MEMS driving electronics are placed inside the cylindrical housing of the MEMS scan head (see Fig. 17). The amplitude and phase control of the MEMS scanning elements uses the PZR sensors integrated on the MEMS chips for position feedback. In closed-loop operation an uncertainty (defined as standard deviation at MSA $=12 \mathrm{deg}$ ) of $0.012 \mathrm{deg}$ was achieved for scan amplitude and $0.035 \mathrm{deg}$ for phase.

The long-term stability of the mechanical scan amplitude and the PZR position signal (measured in open-loop operation over a period of $46 \mathrm{~h}$ ) are shown in Fig 18. An optical autocollimator was used to reference scan angle and phase. Only small deviations of max 0.1 deg were measured in open-loop operation. It is obvious that the angular accuracy is within the total angular budget of $0.2 \mathrm{deg}$, specified for the MSM receiver array of Fovea3D. To prevent temperatureinduced changes in MEMS behavior (e.g., resonance frequency), mechanical decoupling structures are integrated into the MEMS design, which elastically suspend the SOI membrane

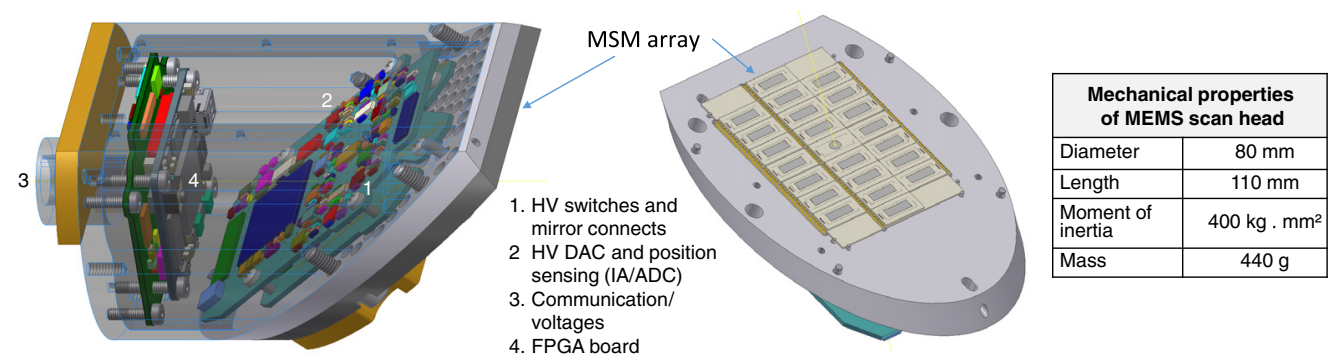

Fig. 17 3D CAD model of the optical MEMS scanning head Fovea3D with hybrid MEMS array and driving electronics.

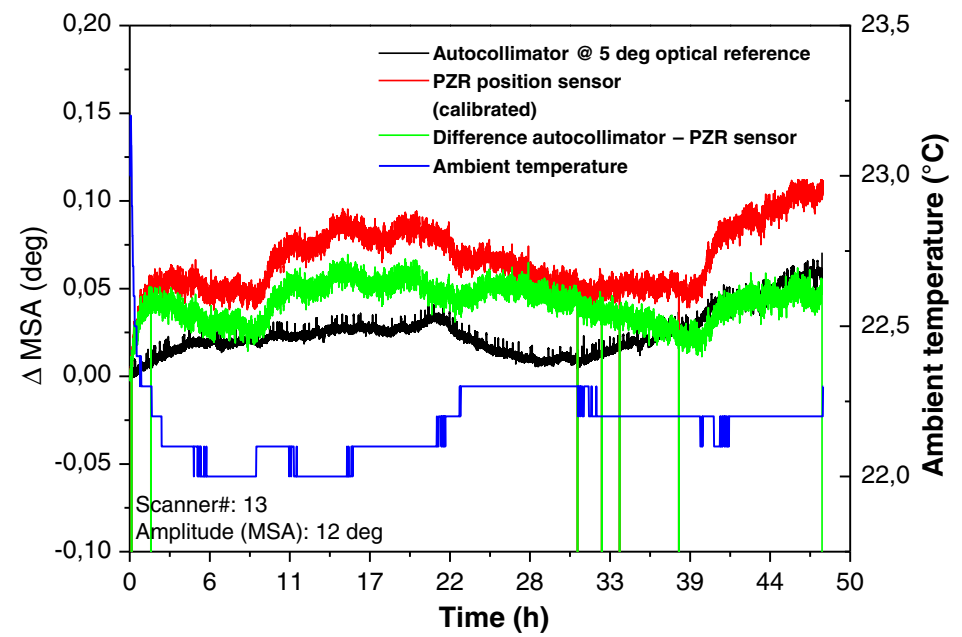

Fig. 18 Experimental results of the hybrid microassembled MSM array: long-term stability of the scan amplitude and PZR position signal. 
containing the movable MEMS elements within the outer chip frame coupled to the environment. This reduces by design the temperature induced changes of resonance frequency by a factor of 10. Details of the mechanical decoupling structures consisting of two degree of freedom springs are shown in Fig. 14(b).

The MEMS scanning head Fovea3D is rotated by an electromagnetic motor. For electrical connection of the rotating MEMS scanning head to the LiDAR system, six electrical slide contacts are used (see Fig. 4).

\subsection{ToF Camera Fovea3D}

The LiDAR system Fovea3D and the final MEMS scan head are shown in Fig. 19. The pulsed ToF distance measuring system uses a fiber amplified laser with $P_{\text {average }}=1.5 \mathrm{~W}, P_{\text {pulse }}=$ $1500 \mathrm{~W}, \lambda=1550 \mathrm{~nm}$, and $1 \mathrm{MHz}$ pulse repetition rate. This pulsed ToF distance measuring module [developed by Fraunhofer Institute for Physical Measurement Techniques (IPM)] achieves a technical performance that exceeds commercially available state-of-the-art systems in several criteria. Pulse repetition rates of up to $2 \mathrm{MHz}$, pulse generation times of a few picoseconds, and standard deviations in ToF measurements of about 45 picoseconds (equal to $6 \mathrm{~mm}$ distance uncertainty) were realized. The latter was caused by about 25 picoseconds attributable to the properties of the commercial timing module used, and 20 picoseconds to the entire signal chain of pulse generation, amplification, analog receiver, and pulse discrimination.

The analog receiver circuit has a wide dynamic range of $>40 \mathrm{~dB}$ (optical power) and $>80 \mathrm{~dB}$ (voltage). This results in an uncertainty of the ToF distance measurement of about $6 \mathrm{~mm}$ for $30-\mathrm{m}$ distance of a target with $10 \%$ reflectance. These are good values that are unique even in their combination. Hence, the Fovea3D camera can capture 3D images at $1 \mathrm{Mpixel} / \mathrm{s}$ or 10 frames at 100 kpixels/s over a $120 \mathrm{deg} \times 60 \mathrm{deg}$ FOV for horizontal oscillation or potentially 360 deg $\times 60$ deg FOV for horizontal rotation using a typical distance measuring rate of $1 \mathrm{MVoxel} / \mathrm{s}$. First 3D images of the 3D ToF camera Fovea3D (using a preliminary scanning system with reduced receiver aperture of $10 \mathrm{~mm}$ instead of the full effective aperture of $23 \mathrm{~mm}$ ) are shown in Fig. 20. Visible is a 3D point cloud with a foveated region within the ROI demonstrating the concept of horizontal foveation used in the Fovea3D system. In this test, both a small ball-pen $(\varnothing<1 \mathrm{~cm})$ and a horizontal wire only 0.5 -mm thick could be clearly detected at a distance of $5 \mathrm{~m}$ within the foveated ROI.

(a)

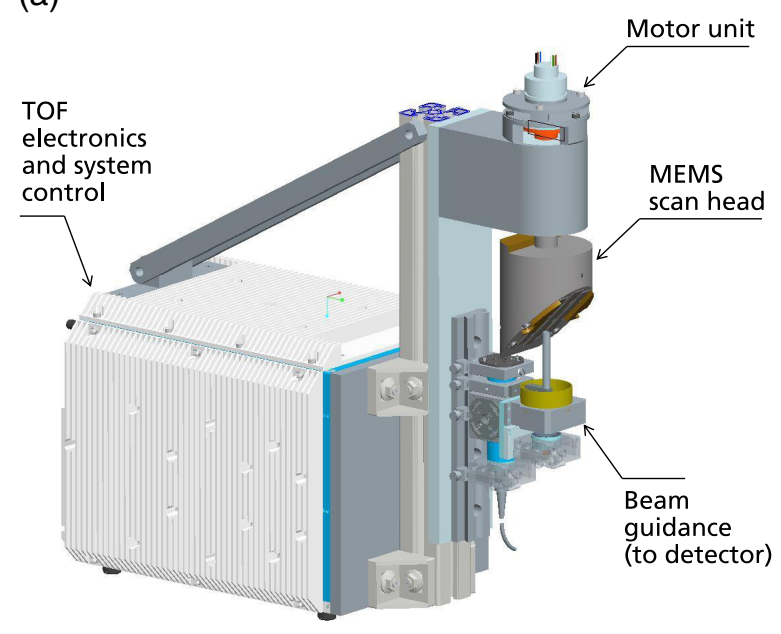

(b)

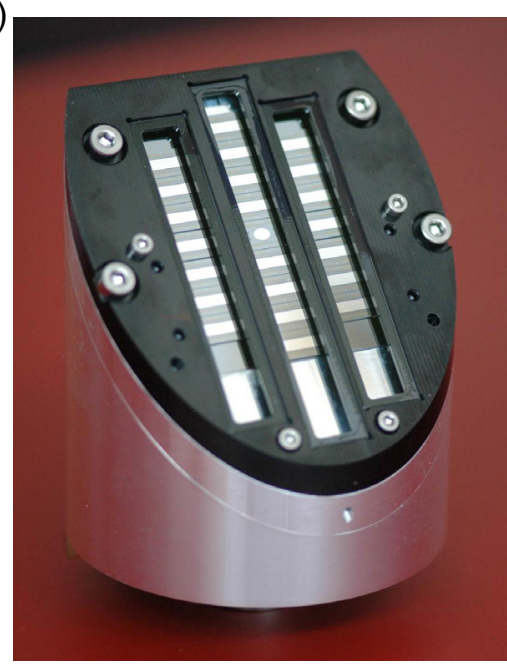

Fig. 19 System integration of hybrid 1D MSM array Fovea3D: (a) 3D CAD model of the pulsed 3D ToF camera (developed by Fraunhofer IPM) with integrated scan head and (b) photograph of the fully assembled MEMS scan head. 
(a)

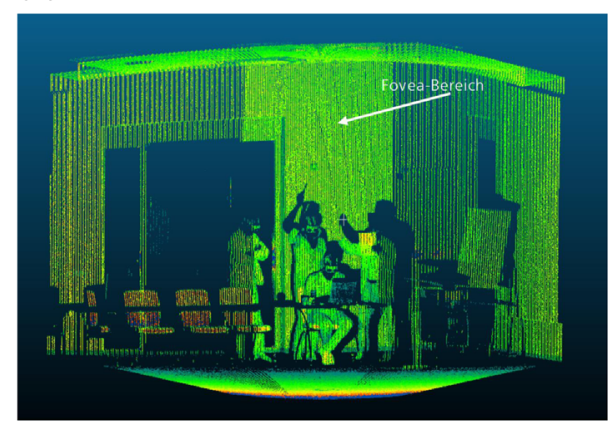

(b)

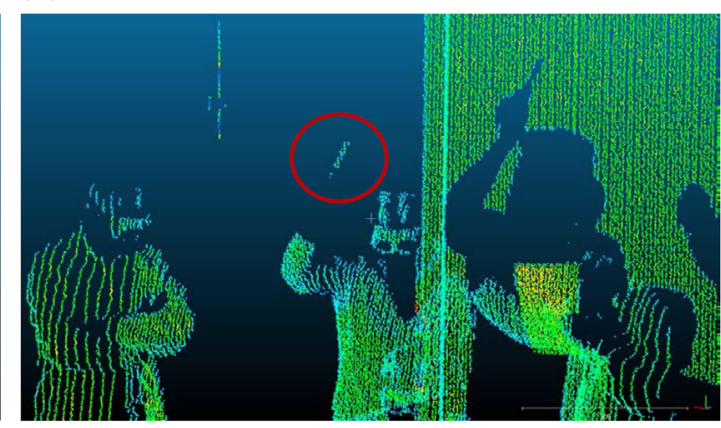

Fig. $203 \mathrm{D}$ images captured with the pulsed ToF distance measurement system of Fovea3D (source Fraunhofer IPM): (a) 3D point cloud with foveation and (b) detail of ROI at $5 \mathrm{~m}$ distance; a small ball-pen ( $<1 \mathrm{~cm}$ diameter) could be clearly detected.

\section{Conclusion}

A compact and fast 1D MEMS scanning module with large aperture was developed and integrated into the panoramic adaptive 3D ToF laser camera Fovea3D for an increased distance measuring range of up to $100 \mathrm{~m}$. The adaptive linearized scanning is realized in the horizontal direction by means of a conventional electromagnetic drive, enabling a 3D ToF camera with video-like image rate and partial foveation properties as required for future outdoor security applications (e.g., monitoring/surveillance of industrial plants and constructions, airports, etc.). Resonant vertical scanning at $1600 \mathrm{~Hz}$ oscillation frequency guarantees the fast bidirectional scanning rate for video-like 3D imaging and a large vertical optical scan range of up to $60 \mathrm{deg}$. To provide the full effective reception aperture of $23 \mathrm{~mm}$ (required for the long-distance ToF camera Fovea3D), 23 single 1D MSM elements, selected by eigenfrequency, were hybrid integrated using a new microassembly technique with self-alignment. A FPGA-based MEMS driving electronics were developed for synchronous operation of all individual receiver MSM elements with respect to the coaxial sending mirror. For the industry-ready resonant MEMS scanner technology of Fraunhofer IPMS, the hybrid concept of Fovea3D allows the best technical compromise of opposite requirements: partial foveation, fast scanning speed, large scan range, enlarged effective aperture of $D_{\text {eff }}=23 \mathrm{~mm}$, high yield, and moderate system costs. The adaptive 3D ToF camera Fovea3D provides a distance measuring rate of $1 \mathrm{MVoxel} / \mathrm{s}$ and a ToF distance measurement uncertainty of $6 \mathrm{~mm}$ at 30-m distance measuring range. This allows, e.g., $3 \mathrm{D}$ images at $1 \mathrm{Mpixel} / \mathrm{s}$ or 10 frames at $100 \mathrm{kpixels} / \mathrm{s}$ over a $120 \mathrm{deg} \times 60 \mathrm{deg}$ (for horizontal oscillation) or (potentially) $360 \mathrm{deg} \times 60 \mathrm{deg}$ (for rotation) large FOV.

\section{Outlook}

The use of MSMs for 3D ToF LiDAR systems is highly topical. ${ }^{11}$ Nowadays, there are many $\mathrm{R} \& \mathrm{D}$ activities and industrial product developments focusing on new applications, especially lower priced miniaturized 3D LiDAR sensors required for high-volume markets, most notably/in particular for automotive applications, such as autonomous driving. ${ }^{8-10}$ To meet the high requirements of automotive applications in terms of cost, miniaturization, and reliability, ${ }^{32}$ MSMs are typically considered only for the laser emitting path of the LiDAR sensor, whereas the receiver optics are realized by nonscanning optics. Frequently, line scanning in combination with optical array detectors is preferred ${ }^{9,33}$ to $2 \mathrm{D}$ single point scanning. Nowadays, solid-state LiDAR systems ${ }^{34-37}$ have to be considered in addition to MEMS-based LiDAR. Nevertheless, the approach of fast scanning 1D MSM arrays for ToF receiver optics presented in this paper is very interesting for sophisticated LiDAR measuring systems (pushbroom scanning ToF systems, e.g., for railroad safety inspection), which require very high scanning speeds. Especially, for highly mobile, movable LiDAR platforms, lightweight, fast scanning systems for scanning receiver optics do not exist. The hybrid assembled MEMS scanner array presented in this paper 
Sandner et al.: System integration of hybrid assembled large aperture Micro Scanner Array...

will be able to increase the scanning speed by a factor of four in comparison with state-of-the-art LiDAR systems used, e.g., for mobile 3D road inspection systems. ${ }^{2}$

\section{Acknowledgments}

The authors would like to thank the Fraunhofer society for their financial support of the presented work within the project "WISA: Fovea-3D Adaptive 3D Recording," which was carried out in collaboration with the Fraunhofer IPM. Here, our special thanks go to our colleagues Claudia Baulig and Stefan Schwarzer from Fraunhofer IPM.

\section{References}

1. P. McManamon, Field Guide to LiDAR, SPIE Press, Bellingham, Washington (2015).

2. A. Reiterer, K. Predehl, and M. Leidinger, "Die Entwicklung von LaserscannernHerausforderungen bei neuartigen Anwendungen," in Terrestrisches Laserscanning, TLS 2017: (DVW-Schriftenreihe 88), pp. 9-21, Wißner, Augsburg (2017).

3. M. Ulbricht, "3D-Luftschadstoffmessungen mit LiDAR," Tech. Mess. 63(7/8), 278-281 (1996).

4. T. Ringbeck, "A 3D time of flight camera for object detection," in Proc. of 8th Conf. Opt. 3D Meas. Tech., Zürich (2007).

5. T. Oggier et al., "SwissRanger SR3000 and first experiences based on miniaturized 3D-TOF cameras," in Proc. 1st Range Imaging Res. Day, pp. 97-108 (2005).

6. C. Niclass et al., "Design and characterization of a $256 \times 64$-pixel single-photon imager in CMOS for a MEMS-based laser scanning time-of-flight sensor," Opt. Exp. 20(11), 11863-11881 (2012).

7. K. Ito et al., "System design and performance characterization of a MEMS-based laser scanning time-of-flight sensor based on a $256 \times 64$-pixel single-photon imager," IEEE Photonics J. 5(2), 6800114 (2013).

8. J. Kernhof, J. Leuckfeld, and G. Tavano, "LiDAR-Sensorsystem für automatisiertes und autonomes Fahren," in Automobil-Sensorik 2, T. Tille, Eds., pp. 29-54, Springer SpringerVerlag, Berlin, Heidelberg (2018).

9. H.W. Yao et al., "MEMS-based LiDAR for autonomous driving," Elektrotech. Inf. 135(6), 408-415 (2018).

10. I. Maksymova et al., "Detection and compensation of periodic jitters of oscillating MEMS mirrors used in automotive driving assistance systems," in Proc. 2019 IEEE Sens. Appl. Symp. (SAS), Sophia Antipolis, pp. 1-5 (2019).

11. D. Wang, C. Watkins, and H. Xie, "MEMS mirrors for LiDAR: a review," Micromachines 11, 456 (2020).

12. U. Hofmann et al., "Resonant biaxial 7-mm MEMS mirror for omnidirectional scanning," J. Micro/Nanolithogr. MEMS MOEMS 13, 011103 (2013).

13. L. Ye, G. Zhang, and Z. You, "Large-aperture kHz operating frequency Ti-alloy based optical micro scanning mirror for LiDAR application," Micromachines 8(4), 120 (2017).

14. F. Senger et al., "A 2D circular-scanning piezoelectric MEMS mirror for laser material Processing," Proc. SPIE 11697, 1169704 (2021).

15. F. Schwarz et al., "Resonant 1D MEMS mirror with a total optical scan angle of $180^{\circ}$ for automotive LiDAR,“ Proc. SPIE 112931129309 (2020).

16. S. T. S. Holmstrom, U. Baran, and H. Urey, "MEMS laser scanners: a review," J. Microelectromech. Syst. 23, 259-275 (2014).

17. A. Fischer et al., "Extension of frequency modulated Doppler global velocimetry for the investigation of unsteady spray flows," Opt. Lasers Eng. 63, 1-10 (2014).

18. H. Schenk et al., "Single crystal silicon micro mirrors," Phys. Status Solidi (c) 6(3), 728-735 (2009).

19. T. Sandner et al., "3D imaging using resonant large-aperture MEMS mirror arrays and laser distance measurement," in Proc. IEEE/LEOS Int. Conf. Opt. MEMS and Nanophotonics, p. 4607837 (2008). 
20. T. Sandner et al., "Large aperture MEMS scanner module for 3D distance measurement," Proc. SPIE 7594, 75940D (2010).

21. T. Sandner et al., "Quasi-static microscanner with linearized scanning for an adaptive threedimensional laser camera," J. Micro/Nanolithogr. MEMS MOEMS 13(1), 011114 (2014).

22. T. Sandner et al., "Microscanner with vertical out of plane combdrive," in Proc. IEEE/LEOS Int. Conf. Opt. MEMS \& Nanophotonics (OMN), pp. 33-34 (2011).

23. D. Jung et al., "Vertical comb drive microscanners for beam steering, linear scanning and laser projection applications," Proc. SPIE 8252, 82520U (2012).

24. R. Schroedter et al., "Microcontroller based closed-loop control of a 2D quasi-static/ resonant microscanner with on-chip piezo-resistive sensor feedback," Proc. SPIE 10116, 1011605 (2017).

25. R. Schroedter, "Modellbasierter Systementwurf zur Steuerung und Regelung quasistatischer Mikroscannerspiegel mit elektrostatischem Kammantrieb," PhD Thesis, Jörg Vogt Verlag (2018).

26. B. Satzer et al., "Micromirror-based sending and detection optical assembly for time-offlight laser scanners," Proc. SPIE 8439, 84390Z (2012).

27. T. Sandner et al., "Hybrid assembled micro scanner array with large aperture and their system integration for a 3D ToF laser camera," Proc. SPIE 9375, 937505 (2015).

28. R. Halterman and M. Bruch, "Velodyne HDL-64E LiDAR for unmanned surface vehicle obstacle detection," Proc. SPIE 7692, 76920 (2010).

29. C. Ataman and H. Urey, "Modeling and characterization of comb-actuated resonant microscanners," J. Micromech. Microeng. 16, 9-16 (2006).

30. J. Grahmann et al., "MEMS-mirror based trajectory resolution and precision enabled by two different piezoresistive sensor technologies," Proc. SPIE 9760, 976006 (2016).

31. T. Sandner et al., "Hybrid assembled MEMS scanner array with large aperture for fast scanning LiDAR systems," Tech. Mess. 86(3), 151-163 (2019).

32. J. Grahmann et al., "Vibration analysis of micro mirrors for LiDAR using on-chip piezoresistive sensor," Proc. SPIE 11293, 1129308 (2020).

33. L. Pohl et al., "Wide angle LiDAR demonstrator based on a resonantly operated 1D MEMS mirror capable of scanning $180^{\circ}$," Proc. SPIE 11697, 116970D (2021).

34. C. M. Liu et al., "Reflex optical phased array for autonomous vehicles using new scheme of LiDAR design," Proc. SPIE 11697, 116970J (2021).

35. R. Nicolaescu et al., "3D imaging via silicon-photonics-based LiDAR," Proc. SPIE 11691, 116910G (2021).

36. G. T. Reed, "Silicon photonics breaks new ground," Proc. SPIE 11691, 1169102 (2021).

37. M. Ding et al., "Silicon photonics nonlinear switch as conditional circulator for singleaperture LiDAR systems," Proc. SPIE 11691, 116910L (2021).

Thilo Sandner studied electrical engineering at the Technical University of Dresden, Germany, where he received his doctorate in 2003. Since 2003, he has been working as a scientist at the Fraunhofer IPMS, where he headed the R\&D group for MSMs for more than 10 years. Currently, he works as a project manager and key researcher for development of MOEMS components, system design, and new applications of photonic microsystems such as MEMS-based LiDAR.

Thomas Graßhoff studied microsystem technology at the University of Applied Sciences Berlin, Germany. He has been working as a MEMS designer at the Fraunhofer IPMS since his graduation in 2005. In 2014, he received his MSc degree in computational engineering from Beuth University of Applied Sciences Berlin. His field of work as a scientist includes the component design of MEMS devices with a focus on FEA simulation of mechanical and multiphysical domains.

Wolf-Dietrich Owe studied electrical engineering at the University of Applied Sciences Dresden, Germany. He has been working as a scientist at the Fraunhofer IPMS since 1992. His work focuses on the lithographic mask layout and reliability tests of microscanning devices.

Andreas Herrmann studied microsystem technology at the University of Applied Sciences Mittweida, Germany. Since 2002, he has been working as a scientist at the Fraunhofer 
IPMS for development of bulk micromachining technologies and MOEMS process integration. In 2011, he received his MSc degree from the University of Cottbus in 2012. His actual work focuses on DRIE process development.

Michael Wildenhain received his Dipl.-Ing. (FH) degree from the University of Applied Sciences Zwickau, Germany, in 1999, and his MS degree from the University of Cottbus, Germany, in 2012. In 2000, he joined at the Fraunhofer IPMS. He has more than 15 years of experience in MEMS micromirror devices for several applications. As a project manager, he heads large industrial projects focusing on product development and preseries production of microscanning devices.

Markus Schwarzenberg studied information technology at the Technical University of Dresden, Germany, where he received his doctorate in 2000 . He has been working as a scientist at the Fraunhofer IPMS since his graduation in 1996. His work focuses on the area of software development, signal processing, and system design of electronic control systems for MEMS devices. Currently, he heads the electronics and software development working group within the IPMS business unit Active Microoptical Devices and Systems (AMS).

Jan Grahmann defended his dissertation, "Highly sensitive resonant gas sensors based on single crystal plate-oscillators," at the University of Chemnitz, Germany, in 2008, and joined at the Fraunhofer IPMS, in 2007. He has been working in the field of micromechanical scanning mirrors and managed international public and industrial projects. In 2010, he became group manager. Since 2013, he has been managing the business unit AMS. 